\title{
INVERSIÓN EN PROGRAMAS SOCIALES SELECTIVOS: RECTORÍA SECTORIAL, RENDICIÓN DE CUENTAS Y EVALUACIÓN DEL DESEMPEÑO COMO FACTORES CLAVE ${ }^{1}$
}

SELECTIVE INVESTMENT IN SOCIAL PROGRAMS: SECTORAL RECTORY, ACCOUNTABILITY AND PERFORMANCE EVALUATION AS KEY FACTORS

\author{
Manuel Antonio Barahona Montero² \\ Ana Cristina Carmona Segnini ${ }^{3}$ \\ Greivin Salazar Álvarez ${ }^{4}$ \\ Joan Pamela Pan Sanabria ${ }^{5}$
}

\begin{abstract}
Resumen
Los tres factores clave que se deben potenciar para mejorar la sostenibilidad de los programas sociales selectivos, su rectoría y gobernanza, el proceso de rendición de cuentas, así como la evaluación del desempeño, misma que tendría que incluir un claro esquema de incentivos asociado a la consecución de resultados y metas. Además de contar con estos tres factores, facilitaría la implementación del ciclo PHVA (Planificar - Hacer - Verificar - Actuar) en tales programas y su marco institucional. Para mejorar esta situación es necesario previamente superar aspectos culturales y legales, propios de los países latinoamericanos.
\end{abstract}

Palabras clave: Programas sociales; sostenibilidad; rectoría; rendición de cuentas; evaluación del desempeño.

doi: http://dx.doi.org/10.15359/eys.20-47.2

Fecha de recepción: 23 de octubre del 2014. Fechas de reenvíos: 27-04-2015. Fecha de aceptación: 21 de mayo del 2015. Fecha de publicación: 28 de junio del 2015

1 Proyecto de investigación 0492-10: Sostenibilidad de la inversión social pública en programas sociales selectivos, Escuela de Economía, Universidad Nacional, Costa Rica.

${ }^{2}$ Catedrático, Escuela de Economía, Universidad Nacional. Costa Rica. mbarahona.montero@gmail.com

${ }^{3}$ Docente e Investigadora, Escuela de Economía, Universidad Nacional. Costa Rica. cris18@racsa.co.cr

${ }^{4}$ Académico, Escuela de Economía, Universidad Nacional. Costa Rica. grsala1@gmail.com

${ }^{5}$ Asistente de Investigación, Escuela de Economía, Universidad Nacional. Costa Rica. jpps08@outlook.com

Manuel Barahona, Ana Cristina Carmona, Greivin Salazar y Joan Pamela Pan 


\section{Abstract}

There are three key factors that must be implemented to improve sustainability of Selective Outreach Programs: management and governance, accountability and performance evaluation; the latter should include a clear incentive scheme related to attaining goals and results. In addition to these three factors, implementing the PDCA (Plan - Do - Check - Act) cycle into such programs and their institutional framework would be helpful. In order to improve this situation, it is necessary to first overcome those cultural and legal aspects typically seen in Latin American countries.

Keyword: Outreach programs; sustainability; governance; accountability; performance evaluation.

\section{Introducción}

Costa Rica sustenta su sistema de protección social en un conjunto de políticas universales, que por medio de una red de servicios para todos los habitantes, se complementan con programas selectivos orientados a la población más pobre y vulnerable. Precisamente, en la perspectiva de contextualizar el universo de estudio en los Programas Sociales Selectivos (PSS), se considera la caracterización de los mismos en su contexto normativo, institucional y programático.

En este sentido, el presenta artículo tiene como objetivo evaluar el estado de la función de rectoría de los programas sociales selectivos, su marco institucional, así como de los procesos de rendición de cuentas y evaluación del desempeño bajo una perspectiva de gobernanza y amparado en el ciclo PHVA, para así delimitar pautas que puedan fortalecer la sostenibilidad de estos programas.

Para tales fines, el documento está integrado por cuatro secciones adicionales a la presente introducción, en la primera se desarrolla el marco teórico-conceptual, es decir se explica en qué consiste el ciclo PHVA y como este será utilizado para concretar el objetivo planteado, en la segunda se contextualizan cuáles son las instituciones e iniciativas que forman parte de los PSS; en la tercera se evalúa los cuatro elementos sustantivos antes mencionados: rectoría, marco institucional, rendición de cuentas y evaluación del desempeño. Por último, en la cuarta sección se desarrollan las conclusiones y recomendaciones.

\section{Contextualización de los PSS}

Por PSS se entiende como el conjunto de programas sociales de carácter selectivo, dirigidos a poblaciones meta específicas en función de algún elemento de vulnerabilidad, las personas en condición de pobreza o riesgo social como los niños, niñas y adolescentes, jóvenes, mujeres y adultos mayores, los indígenas, los inmigrantes, las personas con alguna discapacidad y los indigentes.

2

Manuel Barahona, Ana Cristina Carmona, Greivin Salazar y Joan Pamela Pan

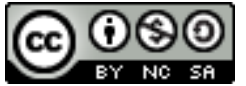

Revista Economía y Sociedad by Universidad Nacional is licensed under a Creative Commons Reconocimiento-NoComercialCompartirlgual 4.0 Internacional License.

Creado a partir de la obra en http://www.revistas.una.ac.cr/index.php/economia 
Respecto a la arquitectura institucional de los PSS, la Contraloría General de la República (2006) determinó que existen instituciones cuyas funciones son exclusivamente de asistencia y promoción social, en tanto que también hay presencia de otras, con fines diferentes, pero que a su vez ejecutan programas con esa misma orientación. En consecuencia, la operación de PSS involucra tanto instituciones completas como programas de instituciones con funciones de vivienda y suministro de agua potable educación, salud y trabajo.

En el primer grupo se identificaron 12 instituciones que ejecutan 29 programas, dentro de los cuales se encuentran dos fideicomisos ${ }^{6}$ y en el segundo grupo se identificaron 10 instituciones que ejecutan 17 programas, entre los cuales también hay dos fideicomisos. Con base en información del Programa Estado de la Nación, la CGR señala que este sector incluye un total de 22 instituciones y 46 programas (cuatro operan a través de la figura del fideicomiso) que ejecutaron en el 2005 CRC 149.750,3 millones. De estas 22 instituciones, existen 12 cuyas funciones están por completo abocadas a la asistencia y promoción social, mientras que las restantes 10 pertenecen a otros sectores, pero cuentan con al menos un programa orientado para tales fines (ver figura 1)
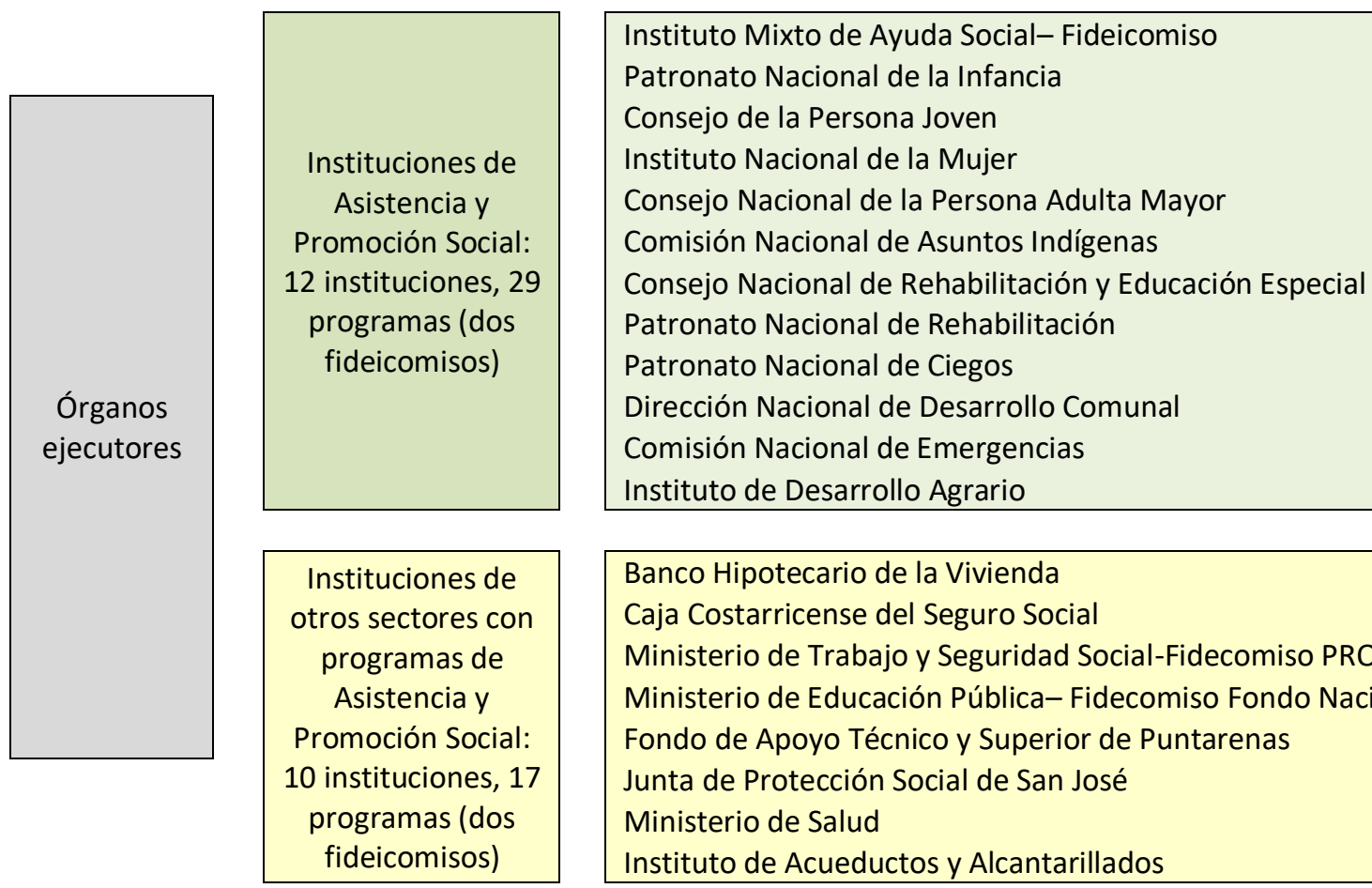

Figura 1. Conformación de los PSS. Fuente: Contraloría General de la República (2006).

\footnotetext{
${ }^{6}$ El fideicomiso es un contrato mediante el cual, una parte transfiere a otra, bienes, dinero o derechos, para que esta los administre y gestione, en procura del logro de los objetivos y fines trazados.
}

Manuel Barahona, Ana Cristina Carmona, Greivin Salazar y Joan Pamela Pan 
Marco teórico

Desde hace algún tiempo el binomio sector público-ineficiencia ha sido "disuelto", el aparato estatal ha dejado de ser visto como aquel mal necesario que deben soportar los procesos de desarrollo de las naciones. Se percibe más bien una preocupación creciente por encontrar la manera de cómo hacer al Estado más eficiente en las diferentes labores en que participa (económicas, sociales, ambientales, regulatorias, entre otros) para que de esta manera se convierta en un efectivo promotor del desarrollo en alianza con la iniciativa privada y de la sociedad civil.

El presente documento comparte este último criterio, por tanto, considera que es factible y necesaria la aplicación de principios de gobernanza a las labores de la hacienda pública similares a las aplicadas en ámbitos privados, en donde se tomen en cuenta las especificidades propias del espacio público.

Para tales efectos, se recurre al ciclo PHVA (Planificar, Hacer, Verificar y Actuar) para evaluar el estado actual de cuatro de los elementos sustantivos para la sostenibilidad de los PSS: la función rectora, el marco institucional, la rendición de cuentas y evaluación de desempeño.

En otras palabras, se evaluará el estado de cada uno de estos elementos, para luego determinar cómo la situación de cada uno de estos impacta la ejecución efectiva del ciclo PHVA en la gestión de las instituciones ejecutoras de los PSS.

\section{Ciclo PHVA}

El ciclo PHVA es una herramienta para la administración y gestión de recursos enfocada hacia la mejora continua, tal como lo indican sus siglas, existen cuatro pasos para alcanzar tal propósito:

- Planificar: se establecen cuáles son las metas que se buscan alcanzar, en qué plazo, las acciones por implementar, así como sus costos y riesgos asociados, por último se definen los responsables.

- Hacer: los responsables ejecutan las acciones encomendadas, en la medida de lo posible, en apego con lo planificado.

- Verificar: como parte de un proceso de control y seguimiento, se coteja qué tan cercanos han sido los resultados obtenidos con respecto a los esperados, en cuanto a su costo, tiempo y alcance.

- Actuar: en función de los resultados del paso anterior, se toman medidas; si se lograron los resultados propuestos de forma eficiente y eficaz se documentan las lecciones aprendidas, en caso contrario se establecen las medidas por aplicar para remediar la situación, según los principios incluidos en el primer paso (Planificar).

De manera ilustrativa, en la figura 2 se muestra el ciclo PHVA y su asociación con los cuatro elementos sustantivos para la sostenibilidad de los PSS.

4

Manuel Barahona, Ana Cristina Carmona, Greivin Salazar y Joan Pamela Pan

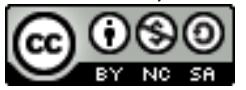

Revista Economía y Sociedad by Universidad Nacional is licensed under a Creative Commons Reconocimiento-NoComercial- 


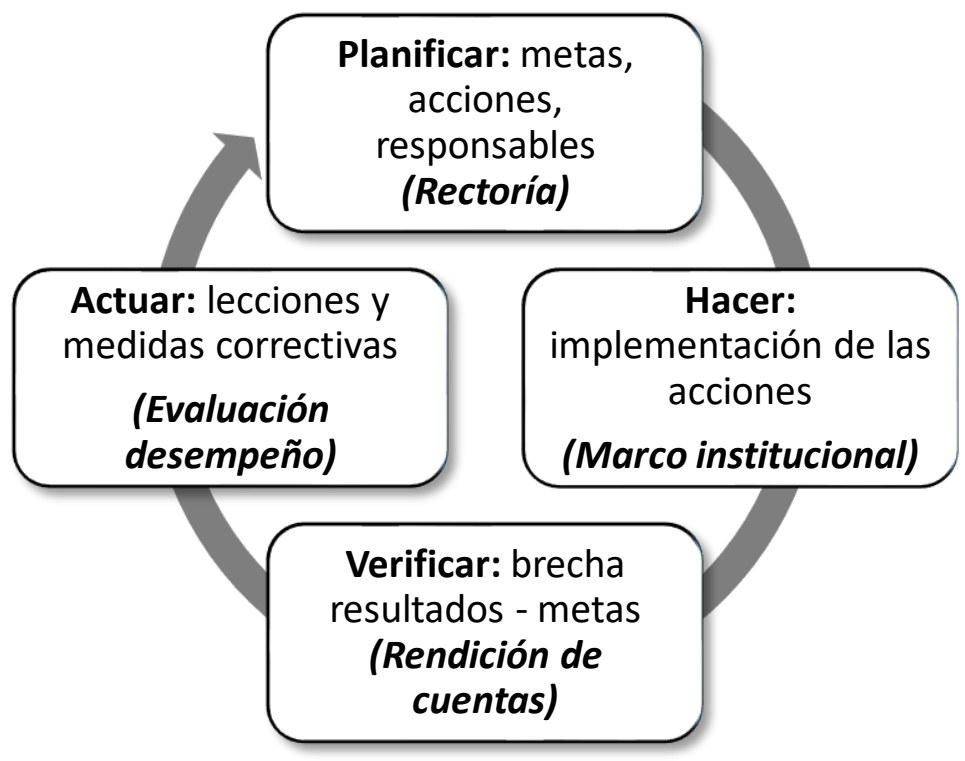

Figura 2. Ciclo PHVA - Planificar, hacer, verificar y actuar. Fuente: elaboración propia.

\section{Evaluación de los elementos sustantivos}

A continuación se evalúan los cuatro elementos sustantivos identificados para la sostenibilidad de los PSS y cómo su situación impacta la implementación efectiva de las actividades de planificar, hacer, verificar y actuar.

\section{La rectoría sectorial}

De acuerdo con los alcances de la Ley de Planificación Nacional (Ley N.5525), este ejercicio corresponde al Ministerio de Planificación y Política Económica (MIDEPLAN) el cual deberá formular, con el apoyo del resto del aparato estatal, el Plan Nacional de Desarrollo (PND), documento donde se establecen metas-país, a partir de las cuales las entidades públicas involucradas realizan su programación, la cual queda plasmada en el Plan Anual Operativo $(\mathrm{PAO})^{7}$, mismo que constituye un documento uniforme, con una estructura básica y una serie de conceptos estandarizados para el sector público. Ha de subrayarse que el ciclo del PND se corresponde estrictamente con el de una administración gubernamental, lo que dificulta un ejercicio de planificación bajo la idea de largo plazo.

\footnotetext{
${ }^{7}$ A nivel interno de las instituciones se debe dar otro proceso de alineamiento, entre el Plan Anual Operativo y el Plan Anual Estratégico.
}

Manuel Barahona, Ana Cristina Carmona, Greivin Salazar y Joan Pamela Pan 
Con este ejercicio se vinculan los planes operativos de cada institución con su respectivo presupuesto y estos a su vez con el PND, con lo que se busca articular un proceso de alineamiento institucional-operativo.

Esta metodología está definida para tres niveles diferentes. En el primer nivel se encuentran las metas sectoriales, las que reflejan el resultado esperado del accionar integral de cada uno de los sectores considerados en el PND; seguidamente se presentan las metas de acciones estratégicas que son las asociadas a todo aquel programa o proyecto cuya ejecución ha sido considerada por la Presidencia de la República de importancia prioritaria, por su impacto en el ámbito nacional, sectorial y regional; por último están las metas institucionales correspondientes con el resultado que se prevé alcanzar mediante la gestión institucional en un período específico. A cada una de estas metas se le define un indicador y una línea base.

Una vez definida esta planificación, entran en juego las llamadas rectorías sectoriales, las que en principio deben velar por la consecución efectiva de las metas país asignadas. En los casos de los PSS, tal como se reseña seguidamente, existe una dificultad histórica para consolidar la figura del ente rector.

En este sentido, los cimientos de la rectoría del sector social en Costa Rica datan del año 1982, durante la administración Monge Álvarez (1982-1986) se creó el Ilamado Gabinete Social, con el objetivo de compensar los efectos sociales adversos generados por la implementación de los programas de estabilización macroeconómica y tratar de recuperar los indicadores de desarrollo humano registrados previo a la crisis de la deuda.

La coyuntura socioeconómica de la época exigió tomar estas medidas: la producción cayó en un $10 \%$ entre 1980 y 1982; el desempleo se duplicó y superó el 14\%; la inflación fue del 90\% en 1982; los salarios reales se redujeron en un $40 \%$; el gasto social cayó de un $23 \%$ del producto interno bruto (PIB) en 1980, a apenas un 16\%; mientras que la pobreza se duplicó (Barahona y Pizk; 2004).

A partir de ese momento la figura de autoridad social fue la constante para las siguientes administraciones, enfocada al tema del combate de la pobreza, pero sin existir una suerte de continuidad y persistencia en este esfuerzo de coordinación.

En la administración Figueres Olsen (1994-1998), mediante el Decreto Ejecutivo N.23310-MP, publicado en La Gaceta N.ㅇ8 del 9de mayo de 1994, se creó el Consejo Social, bajo la dirección de la Segunda Vicepresidencia de la República, figura que fue la encargada de impulsar el llamado Plan Nacional de Combate a la Pobreza (PNCP) ${ }^{8}$. Esta medida significó un primer esfuerzo para formalizarse el concepto de Rectoría Social.

Esta figura fue empoderada, por cuanto el ministro del ramo correspondiente, no era quien definía la política del sector en conjunto con el Ejecutivo, sino que las resoluciones y directrices

\footnotetext{
${ }^{8}$ Este plan estuvo articulado en torno a cuatro ejes: Pro Infancia, Pro Mujer, Pro Trabajo y Desarrollo Local.
} 6 
emitidas por el Consejo del Sector Social eran de carácter vinculante, para este (ministro) y las dependencias e instituciones descentralizadas del área.

La administración Rodríguez Echeverría (1998-2002) no recurrió a la figura de los consejos sectoriales, sino que se inclinó más por un esquema de conducción gubernamental, contó con un solo Consejo de Gobierno, el cual fue el encargado de integrar y articular los diferentes temas de interés.

A nivel instrumental, uno de los cincos pilares que integraron el Plan Nacional de Desarrollo fue el Ilamado "Triángulo de la Solidaridad", definido como "una estrategia que persigue el desarrollo humano duradero, con la participación de todos los costarricenses en programas y proyectos, para beneficio de sus familias y sus comunidades, siendo los protagonistas de sus propios logros, que les permita asumir con apropiación y actitud de triunfo los cambios para el mejoramiento de la calidad de vida" (MIDEPLAN, 2015).

Con esta iniciativa se trató de buscar soluciones en el área social de forma conjunta entre tres actores: las organizaciones comunitarias, los gobiernos locales y las instituciones del gobierno.

En el caso de la administración Pacheco de la Espriella (2002-2006), en el 2002 se creó el Consejo Social y luego en ese mismo año se oficializó el plan "Vida Nueva" para el combate de la pobreza. En su momento, la CGR realizó una autoría operativa a esta iniciativa, en donde señala entre otros aspectos: deficiencias en la formulación; débil correspondencia entre esta iniciativa y el Plan Nacional de Desarrollo; debilidades en la política económica para contribuir a la consecución del objetivo de reducción de la pobreza; inexistencia de retroalimentación entre el Consejo Social y el Consejo Económico sobre temas importantes para la formulación y ejecución del Plan; deficiencias en la definición y aplicación de criterios para la selección de los cantones, distritos y comunidades (Contraloría General de la República, 2004).

Durante la segunda administración Arias Sánchez (2006-2010), nuevamente se volvió a la figura de las rectorías sectoriales. Mediante los Decretos Ejecutivo N.33.151-MP y N.@33.178-MP, organizó en quince sectores la actividad gubernamental, uno de estos correspondió al Sector Social y Lucha contra la Pobreza, cuya rectoría fue encomendada al Ministro de Vivienda y Asentamientos Humanos (MVAH).Incluso, se creó dentro de esta institución el Viceministerio de Desarrollo Social, al cual se le dotó de los recursos financieros y humanos necesarios para ejecutar sus labores, no obstante, a mitad del periodo de gobierno con la salida del ministro y la viceministra, este fue trasladado al Ministerio de Salud, donde posteriormente terminó desdibujándose.

Finalmente, para efectos de la planificación nacional la administración Chinchilla Miranda (20102014) mantuvo los grandes ejes de la sectorialización heredada de la administración precedente, pero se inclinó por la creación de cuatro Consejos Presidenciales, uno por cada uno de los ejes prioritarios y áreas temáticas definidas para su gestión: Ambiental, Bienestar Social 
y Familia (para la atención Integral para el Combate de la Pobreza Extrema, Intervención en Cantones y Comunidades Vulnerables, Red Nacional de Cuido, Educación Técnica e Interconectada, Fortalecimiento del Servicio de Salud), Competitividad e Innovación, Seguridad Ciudadana, y ligó el tema de la dirección superior a la propia Presidencia de la República.

En el caso del Sector de Bienestar Social y Familia se estableció un puesto de ministro sin cartera de Bienestar Social y Familia, ocupado por el presidente ejecutivo del Instituto Mixto de Ayuda Social (IMAS), el cual asumió tareas de coordinación y seguimiento del Consejo Presidencial en la materia.

En suma, al seguir la trayectoria del país en el abordaje de la rectoría en materia social y lucha contra la pobreza se aprecia claramente que la misma no se encuentra plenamente institucionalizada, en consecuencia depende de la administración en turno. Ello ha conducido a la acentuación de fallas en la coordinación interinstitucional, la exacerbación del riesgo de duplicidad programática; el desaprovechamiento de potenciales sinergias entre instituciones y programas (des economías de escala); y la dispersión de los esfuerzos institucionales que impide la atención integral de la población beneficiaria.

Para finalizar esta sección resulta apropiado mostrar el señalamiento realizado en el tema por la CGR en su memoria anual 2011:

Los Programas de Asistencias y Promoción Social, diseñados de forma desarticulada por las instituciones públicas y que han provocado un esquema duplicado de estructuras administrativas para un mismo fin. Esta duplicación de esfuerzos similares en organizaciones separadas, provoca un incremento de costos, tanto para las instituciones públicas involucradas como por los esfuerzos y costos adicionales que deben afrontar los beneficiarios. Cada institución cuenta con unidades administrativas encargadas de los procesos de proveeduría institucional, de asesoría legal, de recursos humanos y de auditoría interna, desaprovechando así las eventuales economías de escala que pudiera tener una organización más integral (Contraloría General de la República, 2012, p. 27).

\section{Marco institucional}

Tal como se explicitó en la segunda sección de este documento, en Costa Rica existe un amplio tejido institucional para la implementación (Hacer) de la política para el combate de la pobreza y la asistencia social, integrado por 22 instituciones y 46 programas.

Desde una perspectiva histórica, con la excepción del Patronato Nacional de la Infancia (PANI - 1930) y el Patronato Nacional de Ciegos (PANACI - 1957), la razón de ser de las primeras instituciones estuvo más orientada a la atención de las necesidades de grupos relativamente amplios y no tan particulares. En 1961 se instauró el Instituto de Desarrollo Agrario (IDA) para el desarrollo de las zonas rurales, en 1962 el Patronato Nacional de Rehabilitación (PANARE) para las personas con algún tipo de discapacidad, en 1967 el

8

Manuel Barahona, Ana Cristina Carmona, Greivin Salazar y Joan Pamela Pan

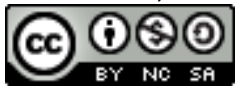

Revista Economía y Sociedad by Universidad Nacional is licensed under a Creative Commons Reconocimiento-NoComercial- 
Dirección Nacional de Desarrollo Comunal (DINADECO) para el desarrollo comunal, en 1969 la Comisión Nacional de Emergencias(CNE) para la atención y prevención de las situaciones de emergencia, en 1971 el Instituto Mixto de Ayuda Social (IMAS) para promover las condiciones de vida de las familias en pobreza, riesgo o vulnerabilidad social.

En cambio, en los últimos 15 años la instauración de instituciones ha respondido más a criterios de género y grupo etario; en 1998 mediante la Ley N. 07801 se creó el Instituto Nacional de la Mujer (INAMU), luego en 1999, con la Ley N. 07935 el Consejo Nacional de la Persona Mayor (CONAPAM) y en 2002 a través de la Ley N. ㅇ261 el Consejo Nacional de la Persona Joven (CNPJ).

En lo que respecta a la antigüedad de estas instituciones, en la década de los treinta del siglo anterior solamente se creó una (PANI - 1930), otra en la de los cincuenta (PANACI - 1957), cuatro en la de los sesenta (IDA - 1961, PANARE - 1962, DINADECO - 1967 y CNE - 1969), tres en la de los setenta (IMAS - 1971, CONAI - 1973 y Consejo Nacional de Rehabilitación y Educación Especial CNREE - 1973), dos en la década de los noventa (INAMU - 1998 y Consejo Nacional de la Población Adulta Mayor CONAPAM - 1999) y otra en la primera década de este siglo (CNPJ - 2002). En consecuencia, la gran mayoría-9 en total, un 75\%-cuentan con 30 o más años de creación, mientras que el restante no superan los 15 años.

Además, la mayor parte de estas instituciones fueron constituidas durante los años sesenta y la primera mitad de los setenta, lo cual coincide con el periodo de mayor apogeo del Modelo Sustitutivo de Importaciones, mismo amparado por un Estado con corte paternalista, benefactor e incluso empresarial.

Por su parte, los PSS financiados por el Fondo de Desarrollo Social y Asignaciones Familiares(FODESAF) presentan una situación similar a la anterior, el 40,7\% (11 en total) de los 27 programas analizados tienen 30 o más años de antigüedad, de estos sobresale el de "Nutrición y Desarrollo Infantil Integral CEN-CINAI" con 63 años, "Proyectos de Vivienda para Personas en Condición de Pobreza" con 56 años, "Atención Integral a Jóvenes en Riesgo Social" con 55 años y "Construcción de Centros Infantiles y Comedores Escolares" con 50 años. Además, solo un 11,1\% tienen entre 20 y 30 años de antigüedad, un 25,9\% entre 10 y 20 años, un $22,2 \%$ menos de 10 años. De igual forma, los programas más antiguos están enfocados a la atención de la población infantil, rural y en situación de pobreza (ver Tabla 1 y Figura 3). 
Tabla 1.

Antigüedad de los PSS financiados por FODESAF.

\begin{tabular}{|c|c|c|c|}
\hline Institución & Programa & $\begin{array}{l}\text { Año de } \\
\text { creación }\end{array}$ & Antigüedad \\
\hline MSP & Nutrición y Desarrollo Infantil Integral (CEN-CINAI) & 1950 & 63 \\
\hline INVU & Proyectos de vivienda para personas en condición de pobreza & 1954 & 56 \\
\hline $\begin{array}{l}\text { Ciudad de los } \\
\text { Niños }\end{array}$ & Atención Integral a Jóvenes en Riesgo Social & 1958 & 55 \\
\hline MOPT & Construcción de centros infantiles y comedores escolares & 1963 & 50 \\
\hline IMAS & $\begin{array}{l}\text { Necesidades básicas: subsidios a familias en situación de pobreza, riesgo o } \\
\text { vulnerabilidad social }\end{array}$ & 1971 & 42 \\
\hline CCSS & Aseguramiento por cuenta del Estado (ACE) & 1973 & 40 \\
\hline MEP & $\begin{array}{l}\text { Programa de Alimentación y Nutrición del Escolar y el adolescente (PANEA: } \\
\text { Comedores Escolares) }\end{array}$ & 1974 & 39 \\
\hline CCSS & Régimen no Contributivo de Pensiones (RNC) & 1975 & 38 \\
\hline MSP & Saneamiento Básico Rural (SANEBAR) & 1975 & 38 \\
\hline ICAA & $\begin{array}{l}\text { Abastecimiento de agua potable a comunidades rurales y urbano- } \\
\text { marginales (AR) }\end{array}$ & 1975 & 38 \\
\hline PANI & Tutela de los Derechos de los Niños, Niñas y Adolescentes & 1975 & 38 \\
\hline BANHVI & Bono Familiar para la Vivienda (BFV) & 1986 & 27 \\
\hline MTSS & Programa Nacional de Apoyo a Micro y Pequeña Empresa (PRONAMYPE) & 1992 & 21 \\
\hline ICODER & Olimpiadas Especiales & 1992 & 21 \\
\hline FONABE & Becas de estudio (Becas) & 1997 & 16 \\
\hline CCSS & Pacientes en fase terminal (PFT) & 1998 & 15 \\
\hline ICODER & Deporte y Recreación & 1998 & 15 \\
\hline INAMU & Promoción y protección derechos de la mujeres & 1998 & 15 \\
\hline CNREE & Pobreza y discapacidad (PD) & 1999 & 14 \\
\hline MTSS & Programa Nacional de Empleo (PRONAE) & 2000 & 13 \\
\hline CONAPAM & Construyendo Lazos de Solidaridad (CLS) & 2001 & 12 \\
\hline IMAS & Avancemos: subsidio a familias pobres con estudiantes en secundaria & 2006 & 7 \\
\hline MSP & Construcción, ampliación, mejoramiento y equipamiento CEN CINAI & 2007 & 6 \\
\hline IAFA & Prevención para el Consumo de Drogas & 2008 & 5 \\
\hline IMAS & Bienestar y Promoción Familiar (BPF) & 2009 & 4 \\
\hline IAFA & Atención adicciones a menores de edad & 2009 & 4 \\
\hline IMAS & Red Nacional de Cuido y Desarrollo Infantil & 2010 & 3 \\
\hline
\end{tabular}

Fuente: elaboración propia

10

Manuel Barahona, Ana Cristina Carmona, Greivin Salazar y Joan Pamela Pan

(c) (i) ()

Revista Economía y Sociedad by Universidad Nacional is licensed under a Creative Commons Reconocimiento-NoComercial- 

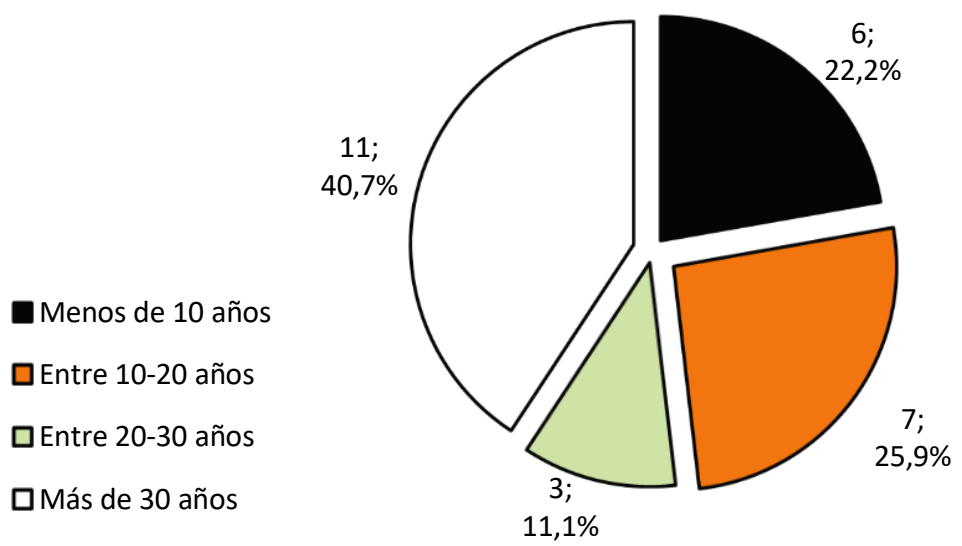

Figura 3. Antigüedad de los Programas Financiados por FODESAF. Fuente: elaboración propia con información de la tabla 1

En síntesis, sí se cuenta con un marco institucional robusto (conjunto de instituciones y programas) dedicado a la asistencia y promoción social de diversos grupos, que incluye los hogares en situación de pobreza y riesgo social, así como la población infantil, joven, adulta, femenina, rural, indígena, en fase terminal, con algún tipo de discapacidad o adicción. El tema crítico es el del alcance y resultados de la gestión de este marco institucional.

\section{Rendición de cuentas y evaluación del desempeño}

Como parte del sector público, las instituciones que conforman los PSS están sujetas a las disposiciones del Sistema Nacional de Evaluación (SINE) cuya creación se remonta a la Administración Figueres Olsen (1994-1998) mediante el Decreto N.24175 del 7 de abril de 1995.

Según el Decreto N. 35755-PLAN del 13 de enero del 2010, mismo que derogó la normativa de cita anterior, el SINE:

Está compuesto por la Presidencia de la República, el MIDEPLAN (quien coordina), el Ministerio de Hacienda, las Unidades de Planificación Institucionales y las rectorías sectoriales a nivel del Poder Ejecutivo en Costa Rica. EI SINE tiene por finalidad la evaluación de la gestión gubernamental en relación con el desempeño institucional, el desarrollo del país y el bienestar ciudadano, por medio de un procedimiento de análisis, monitoreo y evaluación de los resultados de las metas establecidos en el Plan Nacional de Desarrollo (PND) y en otros instrumentos de planificación (Diario Oficial La Gaceta No. 36 del 22 de febrero del 2010; texto subrayado agregado propio).

Una vez articulada la planificación nacional de la administración en curso, posteriormente en un segundo momento y con corte al 30 de junio de cada año, se realiza el seguimiento de cada una de 
las metas, para lo cual se compara el resultado obtenido con la respectiva programación anual, para así determinar su nivel de avance. También, con corte al 31 de diciembre de cada año, se procede con la evaluación de cada una de las metas, para lo que se compara el resultado obtenido con la programación anual y la meta esperada para todo el periodo; en el primer caso se obtiene el nivel de cumplimiento anual mientras que en el segundo, indica el avance acumulado para todo el periodo. A nivel instrumental, este ejercicio es plasmado en la denominada Matriz de Programación, Seguimiento y Evaluación Sectorial e Institucional (MAPSESI).

Por último, para fines de evaluación y de acuerdo con los Lineamientos Técnicos y Metodológicos para la Planeación y Seguimiento a Metas del Plan Nacional de Desarrollo (mayo 2013) emitido por el MIDEPLAN, las metas se clasifican de acuerdo con los siguientes criterios, según su nivel de cumplimiento:

Tabla 2.

Clasificación de las metas según criterio

\begin{tabular}{ll}
\hline Rango de cumplimiento de la meta & Nivel de cumplimiento \\
\hline Igual a $100 \%$ & Meta Cumplida \\
Inferior a $100 \%$ e igual o superior al $80 \%$ & Meta Parcialmente Cumplida \\
Inferior a $80 \%$ e igual o superior a $70 \%$ & Meta Regular \\
Inferior a $70 \%$ y superior a $0 \%$ & Meta Insuficiente \\
Igual a $0 \%$ & Meta No Cumplida. \\
\hline
\end{tabular}

Fuente: MIDEPLAN, 2013, p.21

Además, para el conjunto de Instituciones y Programas Selectivos de lucha contra la pobreza financiados por el Fondo de Desarrollo Social y Asignaciones Familiares ${ }^{9}$ (FODESAF), en el marco del Sistema Nacional de Información Social (SINAIS), se cuenta con un sistema de indicadores de evaluación, mismo que "contiene información mensual de ingresos, gastos, beneficios y beneficiarios, así como indicadores de desempeño, en aspectos como cobertura, resultado, avance, composición, expansión, gasto medio y giro de recursos. (...).Toda esta información está orientada a apoyar la toma de decisiones de políticas públicas, busca transparencia en la administración de estos recursos y la rendición de cuentas a la ciudadanía" (FODESAF: http://www.fodesaf.go.cr/).

Este sistema también requiere de un ejercicio previo de programación, consecuentemente cada uno de los programas deberá definir cuál es el tamaño de la población a la que se espera beneficiar, de cuánto será el gasto por realizar, tanto el total como por beneficiario. Luego,

${ }^{9}$ El FODESAF es el principal instrumento de la política social selectiva en la lucha contra la pobreza; es administrado por la Dirección de Desarrollo Social y Asignaciones Familiares (DESAF) la que a su vez es una dependencia técnica permanente del Ministerio de Trabajo y Seguridad Social.

12

Manuel Barahona, Ana Cristina Carmona, Greivin Salazar y Joan Pamela Pan

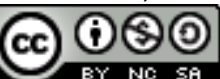

Revista Economía y Sociedad by Universidad Nacional is licensed under a Creative Commons Reconocimiento-NoComercialCompartirlgual 4.0 Internacional License.

Creado a partir de la obra en http://www.revistas.una.ac.cr/index.php/economia 
durante la fase de ejecución, estas cifras son comparadas con los datos efectivos, para así determinar el nivel de avance y cumplimiento alcanzado.

Con este sistema, el evaluador busca encontrar lo siguiente: ¿qué porcentaje de la población objetivo se espera cubrir con el programa?, ¿qué porcentaje de la población objetivo realmente fue cubierta con el programa?, ¿qué porcentaje de la población a la que se esperaba atender fue efectivamente atendida?, ¿qué porcentaje del gasto presupuestado fue ejecutado?, ¿en cuánto creció el gasto total real así como por beneficiario?, ¿qué porcentaje de los recursos que la FODESAF se comprometió a girar a las unidades ejecutoras fueron desembolsados?, entre otras ${ }^{10}$.

Este conjunto de indicadores es un esfuerzo para medir el desempeño de los programas financiados por FODESAF, sin embargo, es necesario introducir algunos otros relacionados más con la productividad de los colaboradores (que de alguna $u$ otra manera intervienen en el proceso de entrega de los beneficios a la población objetivo), así como con el impacto-efecto de los programas.

En esa dirección, se debería medir cuál es la cantidad de personas o núcleos familiares, que en promedio, es atendida por cada uno de los colaboradores de las instituciones encargadas de los PSS, la duración en días que más o menos se tarda desde el inicio de los trámites por parte del interesado(a) hasta que este(a) recibe la ayuda, así como la cantidad de hogares que no están por debajo de la línea de pobreza gracias a la ayuda recibida.

Asimismo, ambos sistemas brindan señales de alerta que pueden y deber ser utilizadas por los entes responsables para establecer las medidas correctivas del caso. Sin embargo, existe un vacío en este proceso de rendición de cuentas, por cuanto no hay un sistema de incentivos vinculado a los resultados obtenidos. Desde luego en el sector público costarricense, al igual que en América Latina (Armijo y Bonnefoy, 2005) los esquemas de remuneración variable en función del logro de objetivos son bastantes escasos, su aplicación es más factible en aquellas empresas estatales que se encuentran en competencia (la banca, los seguros y más recientemente las telecomunicaciones ${ }^{11}$ ).

Por tanto, una parte de la retribución del jerarca de cada institución -a manera de incentivo y no de castigo- debería depender de los resultados alcanzados y el cumplimiento de metas, mismas definidas al más alto nivel del Poder Ejecutivo (ejercicio de forma conjunta entre el MIDEPLAN y el Consejo de Gobierno) y cuyo garante sería la rectoría sectorial.

\footnotetext{
${ }^{10}$ Sobre este último particular, el Anexo muestra, bajo el formato de tabla, los indicadores que conforman este sistema de evaluación, así como su respectivo objetivo y fórmula de cálculo.

${ }^{11}$ El Banco de Costa Rica (BCR), en el Código de Gobierno Corporativo en materia de remuneraciones establece: "la política salarial está orientada a motivar un alto desempeño y enfoque de los empleados hacia la excelencia y productividad, dirigida a atraer y retener el capital humano. Se pueden establecer esquemas de remuneración variable cuando resulten convenientes para el cumplimiento de los fines legales que hayan sido establecidos, los cuales son razonables y proporcionados y aseguran la igualdad al sector para el que se aplica, según se determina en la normativa aplicable" (Banco de Costa Rica; 2009; p. 25; texto subrayado agregado propio).
} 
De este manera, se completaría con el último eslabón del ciclo PHVA analizado previamente; cada jerarca mediante los mecanismos formales de comunicación vertical descendente y la respectiva cadena de mando, le establecería al resto de la organización cuáles son las prioridades (planificar), así esta pasaría a la fase de ejecución (hacer) con un norte plenamente definido para posteriormente informar al jerarca cuáles han sido los resultados obtenidos (verificar) y, en función de estos, establecer si es del caso, las medidas correctivas correspondientes o bien las lecciones aprendidas (actuar) así como los incentivos correspondientes.

En resumen, existe un sistema de indicadores y evaluación del desempeño institucionalizado alrededor del SINE, sin embargo es preciso que este alcance su siguiente nivel, incorpore criterios de eficiencia y productividad, y se establezca algún tipo de "incentivo" hacia aquellas conductas ejemplares que han de emularse.

\section{Conclusiones}

Costa Rica cuenta con un vasto conjunto de políticas universales y programas selectivos dirigidos a mejorar la situación y calidad de vida de la población más pobre y vulnerable, pero hace falta un peldaño: la articulación como tal de un Sistema de Protección Social coherente y robusto. El reflejo de la trascendencia o prioridad que se concede al tema en el país se destaca en que ambos tipos de programas se expandieron en 2010, los universales representaron 58\% de la Inversión Social Pública (ISP) total y el monto destinado a ellos fue de $12,6 \%$ del PIB y $45 \%$ del gasto público; mientras que en los selectivos las cifras fueron de $11,5 \%$ de la ISP total, $2,5 \%$ del PIB y $1 / 10$ del gasto público.

Ahora bien, a pesar de que existen diversos programas (apoyo a la nutrición, formativos, entre otros), aún persisten algunas limitantes importantes para alcanzar mayores niveles de impacto. En esta línea se impone una planificación de largo plazo que logre transcender los esfuerzos particulares de cada administración, plasmados en los "Planes Nacionales de Desarrollo". También destaca la necesidad de resolver el tema de la rectoría de los PSS, dado que hasta la fecha se caracteriza por ser una arena movediza. Además, ha de considerarse que muchos de los programas vienen envejeciendo (datan de los años 70 del pasado siglo), con tendencia a moverse con la inercia del presupuesto histórico.

No cabe duda, entonces que una de las tareas a corto plazo, que traerá resultados en el tiempo, es abordar decididamente el desafío de la rectoría, revisión, innovación y articulación programática. También, falta implementar medidas de impacto en el tema de rendición de cuentas y evaluación del desempeño, en donde se consideren metas definidas de manera realista y a la altura de los retos del desarrollo social.

Para que lo anterior sea posible es preciso superar una serie de limitantes de diferente índole; entre ellas las relacionadas con una cultura institucional rígida y poco perme able a la innovación. En la esfera legal, las disposiciones del servicio civil no permiten estructurar

14

Manuel Barahona, Ana Cristina Carmona, Greivin Salazar y Joan Pamela Pan

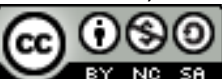

Revista Economía y Sociedad by Universidad Nacional is licensed under a Creative Commons Reconocimiento-NoComercialCompartirlgual 4.0 Internacional License.

Creado a partir de la obra en http://www.revistas.una.ac.cr/index.php/economia 
esquemas salariales con un componente variable en función del logro de metas y objetivos, que premie las conductas destacables, sin castigar aquellas otras no tan deseables, aspecto que desde luego, debe ser objeto de revisión.

Además, los indicadores que se suelen utilizar para evaluar el desempeño se orientan, en lo fundamental, a medir los niveles de ejecución presupuestaria y la cobertura alcanzada, no así los costos de eficiencia, que permita realizar algún tipo de análisis comparativo o benchmarking. De igual manera es importante conceder mayor relevancia al logro o impacto de los programas, en donde se considere su incidencia y resultados en la calidad de vida de la población.

Por último, ha de reiterarse la necesidad de disponer de un sistema de indicadores integrado para la medición y evaluación del desempeño e impacto de los programas sociales selectivos, tarea que evidentemente sería favorecida por el establecimiento de una rectoría programática-institucional robusta.

\section{Referencias}

Armijo, M. y Bonnefoy, J. C. (2005). Indicadores de Desempeño en el Sector Público. (Serie Manual No.45). Recuperado del sitio de internet del Instituto Latinoamericano y del Caribe de Planificación Económica y Social: http://www.cepal.org/es/publicaciones/5611-indicadores-de-desempeno-en-el-sectorpublico

Banco de Costa Rica. (2009). Código de Gobierno Corporativo. San José, Costa Rica.

Barahona, M. y Piszk, S. (2004, agosto 10-12). Aproximaciones y desencuentros en la configuración de una Autoridad Social en Costa Rica: Relato e interpretación de una reforma inconclusa. Seminario Regional de la CEPAL sobre la institucionalidad de la política social. Seminario efectuado en Santiago de Chile.

División de Fiscalización Operativa y Evaluativa. Área de Servicios Sociales, Contraloría General de la República. (2004). Auditoria operativa de la formulación del Plan Social Vida Nueva 2002-2006, de la organización para su ejecución y evaluación y del funcionamiento del Consejo Social de Gobierno durante el periodo 2002-2003 (Informe No. DFOE-SO-22-2004). Recuperado de http://documentos.cgr.go.cr/content/dav/jaguar/sad docs/2004/08759-2004.doc 
División de Fiscalización Operativa y Evaluativa. Área de Servicios Sociales, Contraloría General de la República. (2006). Identificación y Caracterización de los Programas de Asistencia y Promoción Social y Algunas Posibilidades de Mejorar su Integración (Informe No.DFOE-SO-30-2006). San José: Costa Rica.

División de Fiscalización Operativa y Evaluativa Área de Servicios Sociales, Contraloría General de la República, Contraloría General de la República. (2012). Informe sobre la Gestión del Programa de Transferencias Monetarias Condicionada Avancemos (Informe No.DFOE-SOC-IF-10-2012). Recuperado de http://www.asamblea.go.cr/Informes de la_Contraloria/Informes\%202012/k\%20informes noviembre 2012/DFOE-SOC-IF-10-2012.pdf

Ministerio de Hacienda y Ministerio de Planificación y Política Económica (2013). Lineamientos Técnicos y Metodológicos para la Planeación y Seguimiento a Metas del Plan Nacional de Desarrollo; para la Programación Presupuestaria y para el Evaluación Estratégica en el Sector Público en Costa Rica. San José, Costa Rica.

Ministerio de Planificación y Política Económica (2015). El Triángulo de la Solidaridad. Recuperado de http://www.mideplan.go.cr/pnd-1998 2002/Plan19982002/Economico/Triangulo-solidaridad/

Poder Ejecutivo. (2010). Decreto de creación del Sistema Nacional de Evaluación (No. 35755/PLAN). Recuperado del sitio del Diario Oficial La Gaceta: http://www.gaceta.go.cr/pub/2010/02/22/COMP 2202 2010.html\# Toc254337594

16 


\section{Anexo}

Tabla X.

Indicadores del Sistema de Evaluación de los Programas Sociales Financiados por FODESAF

\begin{tabular}{|c|c|c|}
\hline Indicador & Fórmula & Objetivo \\
\hline $\begin{array}{l}\text { Cobertura } \\
\text { Programada } \\
\text { Potencial (CPP) }\end{array}$ & $C P P=\frac{\text { Población Beneficiaria Programada }}{\text { Población Objetivo }} \times 100$ & $\begin{array}{l}\text { Medir el porcentaje que } \\
\text { representa la población } \\
\text { programada a la que llegará el } \\
\text { programa de la población objetivo } \\
\text { pobre. }\end{array}$ \\
\hline $\begin{array}{l}\text { Cobertura } \\
\text { Efectiva } \\
\text { Potencial (CEP) }\end{array}$ & $C E P=\frac{\text { Población Beneficiaria Efectiva }}{\text { Población Objetivo }} \times 100$ & $\begin{array}{l}\text { Medir el porcentaje que } \\
\text { representa la población } \\
\text { efectivamente beneficiaria por el } \\
\text { programa de la población objetivo } \\
\text { pobre. }\end{array}$ \\
\hline $\begin{array}{l}\text { Índice } \\
\text { Efectividad de } \\
\text { Beneficiarios } \\
\text { (IEB) }\end{array}$ & $I E B=\frac{\text { Población Beneficiaria Efectiva }}{\text { Población Beneficiaria Programada }} \times 100$ & $\begin{array}{l}\text { Medir el porcentaje que } \\
\text { representa la población efectiva a } \\
\text { la que llegan los programas de la } \\
\text { población programada. }\end{array}$ \\
\hline $\begin{array}{l}\text { Índice en } \\
\text { Efectividad en } \\
\text { Gasto (IEG) }\end{array}$ & $I E G=\frac{\text { Gasto Efectivo }}{\text { Gasto Programado }} \times 100$ & $\begin{array}{l}\text { Medir el porcentaje del gasto } \\
\text { programado que efectivamente } \\
\text { se ejecutó en el trimestre. }\end{array}$ \\
\hline $\begin{array}{l}\text { Índice } \\
\text { Efectividad Total } \\
\text { (IET) }\end{array}$ & $I E T=\operatorname{Promedio}(I E B$ e $I E G)$ & $\begin{array}{l}\text { Medir de manera conjunta la } \\
\text { eficacia (beneficiarios) y eficiencia } \\
\text { (ejecución presupuestaria). }\end{array}$ \\
\hline $\begin{array}{l}\text { Índice de Avance } \\
\text { Beneficiarios } \\
\text { (IAB) }\end{array}$ & $I A B=\frac{\text { Beneficiarios Ef ectivos } x \text { Trimestre }}{\text { Beneficiarios Programados Anual }} \times 100$ & $\begin{array}{l}\text { Comparar el logro del trimestre } \\
\text { en cuanto a los beneficiarios } \\
\text { efectivos con respecto al total de } \\
\text { beneficiarios programado en el } \\
\text { año. }\end{array}$ \\
\hline $\begin{array}{l}\text { Índice de Avance } \\
\text { Gasto (IAG) }\end{array}$ & $I A G=\frac{\text { Gasto Ef ectivo } x \text { Trimestre }}{\text { Gasto Programado Anual }} \times 100$ & $\begin{array}{l}\text { Comparar el logro del trimestre } \\
\text { en cuanto al gasto realizado con } \\
\text { respecto al gasto total } \\
\text { programado en el año. }\end{array}$ \\
\hline
\end{tabular}

Manuel Barahona, Ana Cristina Carmona, Greivin Salazar y Joan Pamela Pan 
Tabla X (continuación)

\begin{tabular}{|c|c|c|}
\hline Indicador & Fórmula & Objetivo \\
\hline \multirow{2}{*}{$\begin{array}{c}\text { Índice de } \\
\text { Transferencia } \\
\text { Efectiva del Gasto } \\
\text { (ITG) }\end{array}$} & Gasto Efectivo Transferencia Personas & \multirow{2}{*}{$\begin{array}{l}\text { Determinar cuánto del gasto } \\
\text { financiado por el FODESAF al } \\
\text { programa llega efectivamente a } \\
\text { los beneficiarios como } \\
\text { transferencia en dinero. }\end{array}$} \\
\hline & $\begin{array}{c}\text { Gasto Efectivo } \\
\times 100\end{array}$ & \\
\hline \multirow{3}{*}{$\begin{array}{l}\text { Índice de } \\
\text { Crecimiento de } \\
\text { Beneficiarios (ICB) }\end{array}$} & (Beneficiarios Trimestres Actual ) & \multirow{3}{*}{$\begin{array}{l}\text { Comparar el crecimiento } \\
\text { porcentual relativo de los } \\
\text { beneficiarios del programa con } \\
\text { respecto al mismo trimestre del } \\
\text { año anterior. }\end{array}$} \\
\hline & $(\overline{\text { Beneficiarios Trimestre Año Anterior }})$ & \\
\hline & $-1) \times 100$ & \\
\hline \multirow{2}{*}{$\begin{array}{l}\text { Índice de } \\
\text { Crecimiento Gasto } \\
\text { Real (ICG) }\end{array}$} & ( Gasto Real Trimestre Actual ) & \multirow{2}{*}{$\begin{array}{l}\text { Comparar el crecimiento del } \\
\text { gasto real con respecto al mismo } \\
\text { trimestre del año anterior. }\end{array}$} \\
\hline & $\begin{array}{c}I C G=\left((\overline{\text { Gasto Real Trimestre Año Anterior }})^{-1}\right. \\
\times 100\end{array}$ & \\
\hline \multirow{3}{*}{$\begin{array}{l}\text { Índice de } \\
\text { Crecimiento Gasto } \\
\text { Real por Beneficiario } \\
\text { (ICGRB) }\end{array}$} & \multirow{3}{*}{$=\left(\left(\frac{\text { Gasto Real por Beneficiario Año }}{\text { Gasto Real por Beneficiario Año Anterior }}\right)\right.$} & \multirow{3}{*}{$\begin{array}{l}\text { Comparar el crecimiento del } \\
\text { gasto efectivo real por } \\
\text { beneficiario con respecto al } \\
\text { mismo trimestre del año anterior. }\end{array}$} \\
\hline & & \\
\hline & & \\
\hline $\begin{array}{l}\text { Gasto Programado } \\
\text { por Beneficiario } \\
\text { (GPB) }\end{array}$ & $G P B=\frac{\text { Gasto Programado }}{\text { Beneficiarios Programados }}$ & $\begin{array}{l}\text { Mostrar el valor en CRC del } \\
\text { producto programado por } \\
\text { beneficiario por mes. }\end{array}$ \\
\hline Gasto Efectivo por & \multirow{2}{*}{$G E B=\frac{\text { Gasto Efectivo }}{\text { Beneficiarios Efectivos }}$} & \multirow{2}{*}{$\begin{array}{l}\text { Mostrar el valor en CRC que tiene } \\
\text { cada producto entregado según } \\
\text { beneficiario por mes. }\end{array}$} \\
\hline Beneficiario (GEB) & & \\
\hline \multirow{2}{*}{$\begin{array}{l}\text { Índice de Eficiencia } \\
\text { (IEFT) }\end{array}$} & \multirow{2}{*}{$\begin{array}{c}I E F T=\left(\frac{\text { Gasto Programado por Beneficiario }}{\text { Gasto Efectivo por Beneficiario }}\right) \\
\times I E T\end{array}$} & \multirow{2}{*}{$\begin{array}{l}\text { Medir la eficiencia con que se } \\
\text { están utilizando los recursos, lo } \\
\text { cual depende tanto del gasto } \\
\text { realizado por beneficiario como } \\
\text { del grado de ejecución. }\end{array}$} \\
\hline & & \\
\hline \multirow{2}{*}{$\begin{array}{l}\text { Índice de Giro } \\
\text { Efectivo (IGE) }\end{array}$} & \multirow{2}{*}{$I G E=\left(\frac{\text { Ingreso Efectivo Trimestral }}{\text { Ingreso Programado Trimestral }}\right) \times 100$} & \multirow{2}{*}{$\begin{array}{l}\text { Medir el grado de cumplimiento } \\
\text { por parte de la DESAF del giro de } \\
\text { recursos a las unidades } \\
\text { ejecutoras, según lo programado. }\end{array}$} \\
\hline & & \\
\hline \multirow{2}{*}{$\begin{array}{l}\text { Índice de Uso de } \\
\text { Recursos (IUR) }\end{array}$} & $I U R=($ Gastos Efectivos Trimestrales $) \times 100$ & \multirow{2}{*}{$\begin{array}{l}\text { Medir el grado de utilización de } \\
\text { los recursos girados por la DESAF. }\end{array}$} \\
\hline & $I U K=(\overline{\text { Ingresos Efectivos Trimestrales }})^{\times 100}$ & \\
\hline
\end{tabular}

Fuente: FODESAF, en http://www.fodesaf.go.cr/fodesaf/indicadores.html

18

Manuel Barahona, Ana Cristina Carmona, Greivin Salazar y Joan Pamela Pan

(c) (i) (-)

Revista Economía y Sociedad by Universidad Nacional is licensed under a Creative Commons Reconocimiento-NoComercial- 